\title{
EL DIÁLOGO 5+5 Y LA INICIATIVA 5+5 DE DEFENSA
}

\author{
Javier-Ignacio García ${ }^{1}$ \\ UNISCI / IE University
}

\begin{abstract}
Resumen:
El Diálogo $5+5$ es el foro activo de diálogo y cooperación más antiguo que existe entre los países europeos del Mediterráneo Occidental y los países del Magreb. Creado como un mecanismo informal con objetivos modestos, el Diálogo ha incrementado su actividad durante los últimos años en numerosos ámbitos relacionados con la seguridad del Mediterráneo Occidental, particularmente en el campo de la Defensa a través del desarrollo de la "Iniciativa 5+5 Defensa". El artículo analiza las especiales características de estos foros de diálogo y cooperación, sus fortalezas y sus debilidades, así como las posibilidades de desarrollo de estos mecanismos, sus limitaciones y sus perspectivas de futuro.
\end{abstract}

Palabras clave: Dialogo 5+5; Grupo 5+5; Iniciativa 5+5 Defensa; Seguridad en el Mediterráneo; Cooperación Euro-Mediterránea.

Title in English: "The Dialogue 5+5 and the 5+5 Defence Initiative"

\begin{abstract}
:
The "5+5 Dialogue" is the oldest active forum for dialogue and cooperation between Western Mediterranean European countries and the Maghreb countries. Created as an informal mechanism with modest goals, the 5+5 Dialogue in recent years has increased its activity in a significant number of fields related to the security of the Western Mediterranean region, in particular on defense issues, through the development of the so-called "5+5 Defense Initiative". The article analyzes the special characteristics of these initiatives for dialogue and cooperation, their strengths and weaknesses. The possible development of these mechanisms, their limitations, and their future prospects are also studied.
\end{abstract}

Keywords: 5+5 Dialogue, 5+5 Group, 5+5 Defense Initiative, Security in the Mediterranean, EuroMediterranean cooperation.

Copyright (C) UNISCI, 2015.

Las opiniones expresadas en estos artículos son propias de sus autores, y no reflejan necesariamente la opinión de UNISCI. The views expressed in these articles are those of the authors, and do not necessarily reflect the views of UNISCI.

\footnotetext{
${ }^{1}$ El Dr. Javier Ignacio García González es profesor de relaciones internacionales, globalización y ciencias políticas en la Escuela de Comunicación de IE Universtity, es investigador senior de UNISCI y miembro del Foro Hispano-Argelino.

Dirección: Escuela de Comunicación, IE University, Campus de Santa Cruz la Real, C/ Cardenal Zúñiga 12, 40003 Segovia, España

E-mail: javier.garcia@,ie.edu.

http://dx.doi.org/10.5209/rev_RUNI.2015.n39.518[u
} 


\section{Introducción}

La cuenca mediterránea ha sido testigo en los últimos 25 años de no pocos esfuerzos para fomentar el diálogo y la cooperación entre los países que forman parte de ella. Distintas iniciativas que han tenido niveles de ambición y objetivos heterogéneos, tanto en lo que se refiere a los países que formaban parte de ellas - a veces involucrando a prácticamente toda la región, otras solo de alcance subregional-, como respecto a los temas o ámbitos hacia los que se ha querido dirigir esta cooperación -ya sea un diálogo político limitado o cooperación más profunda en aspectos, económicos, sociales o de seguridad-. Desde el originario Proceso de Barcelona lanzado en 1995 hasta la actual Unión por el Mediterráneo² ${ }^{2}$, pasando por la Política de Vecindad de la Unión Europea ${ }^{3}$, todos estos marcos de cooperación han formado parte de un esfuerzo general por crear una "área de paz, de cooperación y de estabilidad" 4 que, por razones tan diversas como los permanentes conflictos en Oriente Medio, las rivalidades regionales o las más recientes "Primaveras Árabes", no han llegado en la mayor parte de los casos a alcanzar los objetivos previstos en el momento de su puesta en marcha.

En este conjunto de iniciativas de diálogo y cooperación que se han lanzado entre los países europeos y los del sur del Mediterráneo desde los años 90, se situará el denominado Foro para el Diálogo en el Mediterráneo Occidental, más conocido como Diálogo $5+5$ o Grupo 5+5. Considerado como el "marco más antiguo de encuentro entre países de la cuenca mediterránea" ${ }^{5}$, el Diálogo aparecerá como un mecanismo que, con sus vaivenes, ha perdurado hasta la actualidad y ha mantenido e incluso aumentado su actividad y los ámbitos de cooperación en los que se ha centrado a lo largo del tiempo, muchos de ellos directa o indirectamente relacionados con la seguridad de esta región, entendida desde el actual punto de vista amplio y multidimensional de la seguridad.

Desde su propósito inicial en 1990 de "reforzar el diálogo político y promover la concertación,...el desarrollo económico, cultural y social en el Mediterráneo occidental" ${ }^{6}$ (con unos ámbitos de cooperación muy generales en Economía, Recursos Humanos y Recursos Naturales $^{7}$ ), el Diálogo $5+5$ ha evolucionado hasta convertirse en un foro de diálogo y

\footnotetext{
${ }^{2}$ El Secretariado de la Unión por el Mediterráneo tiene su sede en Barcelona. Sobre su actividad más reciente ver UfM Secretariat (2015): Unión for the Mediterranean, Activity Report 2014, Barcelona, en http://ufmsecretariat.org/wp-content/uploads/2015/04/Report_web_FINAL.pdf.

${ }^{3}$ Sobre la Política de Vecindad de la Unión Europea ver en este mismo número Guinea Llorente, Mercedes : "La política Europea de Vecindad hacia el Mediterráneo (2003-2015)".

4 "Déclaration commune des neuf pays de la Méditerranée occidentale, sur la coopération et le dialogue en Méditerranée occidentale entre les pays de l'Union du Maghreb arabe et les pays de l'Europe du Sud

Rome le 10 octobre 1990", p. 1. en http://www.iemed.org/observatori-es/recursos/documents/documentsoficials/declaracio-constitutiva-del-dialeg-5-5.

${ }^{5}$ Baba, Marie y Gounon, Claire (2013): Le Dialogue 5+5, Dossier de Prèsentation, FMES, p. 3, en http://fmesfrance.org/ medias/document/DOSSIER $\% 205+5$.pdf.

6 "Déclaration commune des neuf pays de la Méditerranée occidentale, sur la coopération... ", op. cit., p. 1.

${ }^{7}$ Ibid. p. 2. Concretamente, el texto de la Declaración señala:

"Les neuf pays ainsi que Malte ont décidé de promouvoir le dialogue et la concertation en vue de contribuer à la solution des questions politiques et de sécurité d'intérêt commun. Ils ont décidé en outre de développer leur coopération sur une base équilibrée mutuellement avantageuse dans les domaines prioritaires suivants :
}

- A) Economie : - Promotion des échanges commerciaux (dont la coopération en matière des normes de contrôle des produits et des systèmes de certification). - Relations industrielles. Partenariat et investissements. Energie. Transport. Agriculture et autosuffisance alimentaire. Tourisme et artisanat. Transfert technologie.

- B) Ressources humaines : - Affaires sociales et en particulier l'immigration. Education et formation. Recherche scientifique. Communications. Affaires culturelles et sauvegarde du patrimoine. Activités sportives. 
cooperación multidimensional con una agenda de discusión muy amplia, que no sólo abarca las reuniones de los Ministros de Asuntos Exteriores (con temáticas también muy amplias), sino también de los departamentos de Interior, Inmigración, Transporte, Defensa, Turismo, relaciones interparlamentarias, Educación e Investigación, Medio Ambiente y Energía, Agricultura y Seguridad Alimentaria, además de otros asuntos más específicos. Un catálogo temático extenso, para una cooperación con unas características muy determinadas, que han convertido al Diálogo en lo que el embajador Busquets define como "un eslabón esencial de la cooperación Euro-Mediterránea" ${ }^{8}$.

De particular importancia desde la perspectiva de la seguridad será el desarrollo del Diálogo $5+5$ en su dimensión de Defensa. Pese al carácter extremadamente sensible de estas cuestiones, que históricamente han sido fuente de controversias y desconfianza tanto en las relaciones norte-sur del Mediterráneo como en las relaciones sur-sur, la colaboración en algunos temas de Defensa se ha abierto camino con cierto éxito a través de este formato, convirtiéndose la denominada "Iniciativa 5+5 Defensa" en uno de los ámbitos de cooperación que ha mostrado mayor continuidad y actividad desde su lanzamiento en el año 2004.

Será en este contexto donde surgirán toda una serie de preguntas sobre el Diálogo $5+5$ en general, y sobre la Iniciativa 5+5 Defensa en particular, cuyas respuestas pueden contribuir a explicar algunas claves sobre la utilidad de este modelo de cooperación, su potencialidad, limitaciones y las posibilidades de aplicación en otros ámbitos relevantes para la seguridad de esta subregión del Mediterráneo: ¿Cuáles son las razones que han permitido la permanencia y continuidad en el tiempo del Diálogo 5+5 y de la Iniciativa 5+5 Defensa? ¿Cuáles son las características que les han hecho tener cierto grado de éxito, dentro de su modestia? ¿Son sus actividades adecuadas? ¿Se puede potenciar aún más? ¿Podrían ampliarse sus miembros? ¿Cuáles son sus posibilidades de cara al futuro? ¿Son estos foros adecuados como base para iniciativas mucho más ambiciosas? ¿Pueden servir de modelo? ¿Puede sustituir a otras iniciativas o marcos de cooperación fracasados o bloqueados? ¿Qué puede aportar en el marco de la reconfiguración de las relaciones entre los países del norte y del sur del Mediterráneo occidental?

Estas y otras preguntas intentarán ser abordadas a continuación, partiendo de una hipótesis general: el Diálogo 5+5 como marco, y la Iniciativa 5+5 Defensa en particular, tienen continuidad y se consideran útiles para sus participantes por una serie de características muy específicas de este foro que lo han hecho adaptable a las necesidades y la voluntad de cooperación de sus miembros en cada momento. En este sentido, se intentará analizar -a la luz del desarrollo de la iniciativa en los últimos años-, si una posible potenciación de este foro, incluso alterando estas características esenciales, es posible o deseable. Dicho de otra forma, hasta que punto unos cambios en el Diálogo (o en la Iniciativa de Defensa) que puedan suponer un incremento en el número de miembros, en los temas de los que se ocupa, o en el nivel de compromiso en esta cooperación serían convenientes o, por el contrario, sus eventuales consecuencias lo desaconsejan. En suma, si el modelo es útil como es, si es susceptible de cambios y hasta dónde puede llegar su efectividad como instrumento de diálogo, cooperación o integración regional.

- C) Ressources naturelles : - Environnement (et en particulier la lutte contre la pollution, la désertification et les fléaux naturels). Protection civile. Préservation et sauvegarde des ressources halieutiques.

${ }^{8}$ Busquets, Gabriel (2014): "Regional Integration in the Western Mediterranean: the AMU and 5+5", Mediterranean Yearbook 2014, IEMed, Barcelona, p. 90. 


\section{El Diálogo 5+5 como marco de referencia}

Concebido a partir de una idea del presidente francés François Mitterrand a principios de los años $80^{9}$, el Dialogo 5+5 se puso en marcha efectivamente en Roma en 1990, con el objetivo de establecer un proceso de cooperación subregional limitado al Mediterráneo occidental. Se trataba de un foro de diálogo informal sobre cuestiones políticas, limitado a diez países miembros: España, Francia, Italia, Malta y Portugal en la ribera norte, y los cinco países de la Unión del Magreb Árabe por parte de la ribera sur, es decir, Argelia, Libia, Marruecos, Mauritania y Túnez.

Tras unos comienzos que se podían calificar de prometedores ${ }^{10}$, el Diálogo prácticamente se paralizó en los 90 debido a una coyuntura internacional y regional difícil (sanciones contra Libia por el caso Lockerbie en 1992, Guerra del Golfo, tensión Marruecos/Argelia, guerra civil en Argelia etc.), volviéndose a reactivar con nuevas energías con ocasión de la conferencia ministerial de Lisboa de 2001, una vez que se suspenden las sanciones a Libia tras la entrega en 1999 de dos ciudadanos libios para ser juzgados por el atentado de Lockerbie, y la cumbre de Túnez de 2003, una vez que el Consejo de Seguridad de Naciones Unidas levantó las sanciones. La iniciativa retomaba su marcha en un momento oportuno, alejándose de los problemas y conflictos de Oriente Medio que bloqueaban las grandes iniciativas de cooperación multilateral, y con una flexibilidad en su funcionamiento, que se deriva de su carácter informal y de la posibilidad de llevar a cabo en su seno un diálogo amplio y abierto entre los participantes, algo que los expertos siempre han relacionado con su éxito ${ }^{11}$.

Desde entonces, durante los últimos 15 años, el Diálogo $5+5$ ha ido avanzando progresivamente, no sin dificultades como las provocadas en 2011 por las revueltas de la denominada "Primavera Árabe" en Túnez y Libia, que se han ido superando o, al menos, aislando para que no paralizara el proceso de nuevo. A lo largo de los años el Diálogo se ha ido transformado desde sus orígenes casi exclusivamente como foro de diálogo político a una más compleja, un foro regional de cooperación multidisciplinar, sobre un número de temas crecientes $^{12}$. Ese carácter informal al que hacíamos referencia, que supone la no existencia de estructuras permanentes ni la vocación de $\operatorname{crearlas}^{13}$, proporciona a este foro la también citada flexibilidad que le permite tomar formas diferentes según los temas que trate, impulsando programas de acción concretos y adaptándose a las prioridades que marcan los miembros que participan $^{14}$, lo que se traduce en una relación de cooperación respetuosa y de igual a igual.

Si bien las reuniones periódicas de Ministros de Asuntos Exteriores son un eje central del Diálogo, otras citas de alto nivel dan muestra del desarrollo de la iniciativa. Dos Cumbres de Jefes de Estado y de Gobierno han respaldado decisivamente el proyecto (Túnez en 2003 y

\footnotetext{
${ }^{9}$ La idea original consistía en reunir a seis países del Mediterráneo Occidental (Francia, Italia, España, Tunez, Argelia y Marruecos) de forma regular para discutir de asuntos de interés común y como muestra de solidaridad en la región mediterránea, sin remplazar los instrumentos de solidaridad existentes entre los distintos países; ver Busquets, Gabriel (2014): "Regional Integration in the Western... op. cit., pp. 88-89. Ver también, Baba, Marie y Gounon, Claire (2013): Le Dialogue 5+5... op. cit., p. 16.

${ }_{10}^{10}$ Busquets, Gabriel (2014): "Regional Integration in the Western... op. cit., p 89.

11 Ver Ben M'Barek, Slaheddine (2009): "La cooperation en Mediterranee occidentale: 5+5. Bilan et perspectives". Conflictos regionales y estrategias de seguridad, VII Seminario Internacional sobre Seguridad y Defensa en el Mediterráneo, Barcelona, CIDOB, p. 50, en http://www.cidob.org/media2/publicacions/monografias/vii_seminario_seguridad_defensa/08_ben

${ }_{12}$ IEMed (2014): "Chronologies", en Mediterranean Yearbook 2014, IEMed, Barcelona, pp. 373-375.

${ }^{13}$ Coustillière, Jean François (2012) : "L'initiative 5+5 face au nouveau contexte en Méditerranée occidentale", FOCUS article, Observatory of Euromediterranean Policies -IEMedObs-, p. 3, en http://www.iemed.org/observatori/arees-danalisi/arxius-adjunts/copy_of focus/92-Coustiliere.pdf

${ }^{14}$ Ben M’Barek, Slaheddine (2009): "La cooperation en Mediterranee...", op. cit., p. 50.
} 
Malta en 2012) y reuniones ministeriales en otros ámbitos se han ido convirtiendo en habituales ${ }^{15}$; de ministros del Interior (desde 1995), sobre migraciones (desde 2002), relaciones inter-parlamentarias (desde 2003), defensa (desde 2004), turismo (desde 2006), transportes (desde 2007), educación (desde 2009), medio ambiente y energías renovables (desde 2010), agua (2013) o educación superior e investigación (desde 2013). A ellas hay que añadir las reuniones de expertos de alto nivel en otros temas como agricultura o juventud, las frecuentes y numerosas reuniones técnicas de expertos sobre cuestiones y proyectos más específicos y los dos Foros Económicos del Diálogo 5+5 celebrados en 2013 y 2014, donde además de los ministros de Asuntos Exteriores se han dado cita representantes de la sociedad civil, de las asociaciones patronales de los miembros y representantes de grandes empresas y del mundo de los negocios de todos estos países.

La celebración de estos foros ha sido considerada por algunos como un nuevo peldaño en la concertación o integración regional, "una verdadera cooperación reforzada" ${ }^{16}$, que incorpora participantes del sector privado y del mundo empresarial y de negocios. Con todos estos encuentros, según señala Florensa, "los países miembros del $5+5$ han ido tejiendo un foro informal de diálogo subregional para identificar los ámbitos de interés compartido y fomentar las relaciones entre la Unión Europea y la Unión del Magreb Árabe" ${ }^{17}$. Se trata por tanto de un foro que, en su opinión, tendría más capacidades de transformación y de integración que otras iniciativas existentes o por desarrollar en otras zonas del Mediterráneo, ya que el Diálogo podría servir de precedente, de modelo e incluso transformarse en un mecanismo permanente de cooperación reforzada en el Mediterráneo. Si bien ese carácter modélico de algunas de las acciones del Diálogo han sido consideradas como un modelo de cooperación regional $^{18}$, una eventual transformación en un mecanismo permanente, en otras palabras, avanzar en la institucionalización del Diálogo, no es compartido por muchos autores $^{19}$, que ven en ese carácter informal y en su flexibilidad algunas de las razones fundamentales de su éxito (aunque sea limitado) y su perdurabilidad.

Por otra parte, ya desde su propio diseño el Dialogo $5+5$ nacía con un carácter limitado en su alcance, que nunca se ha querido configurar como una alternativa o sustitución de ninguna de las iniciativas de partenariado o asociación más amplias que se han lanzado en el Mediterráneo en las últimas décadas ${ }^{20}$, de las que la Unión por el Mediterráneo o la Política de Vecindad de la UE han sido las más importantes en los últimos tiempos. Por el contrario, el Diálogo en los distintos campos en los que se produce ha sido considerado siempre por sus miembros como un foro complementario, un mecanismo de cooperación "facilitador" de las relaciones entre sus miembros y que no competía con otras iniciativas de mayor ambición. Así, la experiencia en el $5+5$ contribuye a reforzar la posición y las relaciones de confianza tanto en los miembros europeos como africanos, reforzando con ello la presencia y el papel de estos países en otros procesos de integración y concertación. Quizá por ello, con alguna exageración, dadas las características de este diálogo, señala Busquets ${ }^{21}$ que se ha convertido en el "foro de diálogo y cooperación que crea el mayor nivel de consenso entre los socios del sur".

\footnotetext{
${ }^{15}$ Ver Economic Forum of the Mediterranean (2014): 5+5 Dialogue-Chronology of the main meetings (20032013), p. 2 en http://westmediterraneanforum.org/wpcontent/uploads/2013/09/131017 chronology5+51.pdf.

${ }^{16}$ Florensa, Senén: "Mecanismo mediterráneo de cooperación reforzada", AFKAR/IDEAS, invierno 2013/2014, p. 32.

17 Ibid.

${ }^{18}$ Busquets, Gabriel (2014): "Regional Integration in the Western...", op. cit., p 90.

${ }^{19}$ Coustillière, Jean François (2012) : "L’initiative 5+5 face au nouveau contexte...", op. cit., p. 3.

${ }^{20}$ Busquets, Gabriel (2014): "Regional Integration in the Western...", op. cit., p 90.

${ }^{21}$ Busquets, Gabriel (2014): "Regional Integration in the Western...", op. cit., p 85.
} 
Ese carácter limitado no se refiere sin embargo a los posibles ámbitos de cooperación del formato $5+5$. Si hemos visto como a lo largo de los años los campos de trabajo común se han ampliado, no hay razón para pensar que la expansión del Diálogo en este sentido ha llegado a su fin. El límite no está definido en ningún sitio y lo encontramos exclusivamente en la capacidad de los miembros para encontrar intereses compartidos y para coincidir en las prioridades que tienen en cada caso. En el caso específico de la seguridad, una eventual confluencia entre las agendas actuales de los distintos participantes europeos y norteafricanos del Diálogo, que ya se producen o se pueden producir en temas como la inmigración ${ }^{22}$, el crimen organizado $^{23}$, el terrorismo ${ }^{24}$, los problemas medioambientales ${ }^{25}$, la seguridad marítima, la energía ${ }^{26}$, el agua, etc. puede implicar sin duda un incremento en el diálogo entre los países miembros y en la puesta en marcha de medidas e iniciativas concretas de colaboración en políticas sobre estos temas ${ }^{27}$.

Esto será en buena medida posible por otra de las características del Diálogo, su carácter pragmático y orientado a las medidas concretas de colaboración, con una aproximación más técnica que política en todo el proceso ${ }^{28}$. Pese a la mayor visibilidad de las cumbres y conferencias ministeriales, la mayor actividad del Diálogo tiene lugar a nivel técnico, en iniciativas y proyectos concretos que varios miembros identifican como de interés común y con una política de geometría variable en la participación, a la que nadie está obligado. Esto permite mucha mayor flexibilidad y menos limitaciones a la hora de encontrar las áreas de interés común, tanto en iniciativas con miembros del norte y del sur, como en otras solamente entre los miembros del sur.

El relativo éxito de las actividades del Diálogo en sus distintos ámbitos - aunque unos están más desarrollados que otros por su propia evolución y naturaleza-, tampoco está exento de riesgos. Dos son los principales dilemas o debates en los que el Diálogo $5+5$ puede verse envuelto y que podrían transformar el futuro de este foro ${ }^{29}$.

En primer lugar está la cuestión de una eventual ampliación del Diálogo a otros países de la región. El éxito del formato, que funciona en la práctica con "geometría variable" y gran flexibilidad en los participantes, parecería permitir una extensión a otros países del área, como podría ser Egipto u otros países africanos o europeos ${ }^{30}$ que se quisieran incorporar, no faltando voces en este sentido ${ }^{31}$. Sin embargo, eso supondría atentar contra una de las características esenciales del Diálogo, su condición de foro específicamente subregional

\footnotetext{
${ }^{22}$ Sobre el tema de las migraciones en el Mediterráneo occidental ver en este mismo número de la revista: Alternativas a la política migratoria de la Unión Europea en el Mediterráneo occidental.

${ }^{23}$ Sobre el crimen organizado en el Mediterráneo occidental ver en este mismo número de la revista: Hacia un nuevo "flanco sur" en el Gran Magreb.

${ }^{24}$ Sobre el fenómeno terrorista en el Mediterráneo ver en este mismo número de la revista: La Unión Europea y el nuevo terrorismo islamista.

${ }^{25}$ Sobre los problemas medioambientales en el Mediterráneo ver en este mismo número de la revista: Las consecuencias del cambio climático en el Magreb.

${ }^{26}$ Sobre el tema de la energía en el Mediterráneo occidental ver en este mismo número de la revista: Nuevos planteamientos en la seguridad energética de la Unión Europea: opciones e implicaciones en el Mediterráneo occidental.

${ }^{27}$ Como por ejemplo la Estrategia para el agua, aprobada en marzo de 2015.

${ }^{28}$ Coustillière, Jean François (2012) : "L’initiative 5+5 face au nouveau contexte...", op. cit. , p. 4.

${ }^{29}$ Ibid., p. 7.

${ }^{30}$ Egipto y Bélgica quisieron ser incluidos como miembros en el lanzamiento de la iniciativa; ver Poupel, Jean Charles y Garcier, Stefan: "Le dialogue 5+5 et sa problematique", en FMES (2014): Le dialogue 5+5: levier au service d'une reconfiguration des relations euroméditerranéennes. Colloque Octobre 2014, Les Presses du Midi, Marseille, p. 45.

31 Ver Awad, Ibrahim: "Élargissement et duplication du «modele» dialogue 5+5", en FMES (2014): Le dialogue 5+5: levier au service... " , op.cit., pp. 120-124.
} 
circunscrito al área del Mediterráneo occidental y sus problemas e intereses compartidos. Si bien la colaboración con otros foros de mayor alcance no sólo es posible, sino que incluso ya se produce por ejemplo con la Unión por el Mediterráneo, una eventual ampliación de los miembros "titulares" del Diálogo (tanto en el norte como en el sur) muy probablemente lo desnaturalizaría, complicaría su gestión, lo aproximaría a otras iniciativas con las que acabaría colisionando y, muy probablemente, al final provocaría el desinterés de los miembros. Como señalaba una responsable diplomática francesa a finales de 2014, "nosotros deseamos que el $5+5$ siga siendo un espacio limitado, flexible e informal" ${ }^{\prime 32}$, siendo su tamaño limitado una de las características que favorece el diálogo y la discusión de los distintos temas.

La segunda cuestión es una posible mayor institucionalización de este foro o parte de sus iniciativas, que algunos reclaman para aprovechar su nivel de éxito, garantizar su continuidad y operatividad y permitir su crecimiento ${ }^{33}$. Si bien estos aspectos efectivamente podrían verse mejorados con un cierto tipo de estructura formal e institucional, también se correría el riesgo de que esto introdujera cierta rigidez en el proceso, afectara al tipo de relaciones entre los miembros -ahora de igual a igual-, a la confianza mutua entre los participantes -ahora colaborando sin instituciones intermedias-, y al nivel de corresponsabilidad que todos ellos han mostrado con los distintos aspectos del Diálogo ${ }^{34}$-que podrían hacer descansar en la estructura creada, sin que esto fuera necesariamente bueno-. En suma, en tanto estas medidas pudieran afectar negativamente al papel esencial del Diálogo como instrumento de fomento de la confianza, se debería ser muy cauteloso con cualquier desarrollo en este sentido.

\section{La Iniciativa 5+5 Defensa:}

\subsection{Lógica y evolución de una iniciativa modesta, pero factible}

La denominada "Iniciativa 5+5 Defensa" nace en el año 2004, como parte del Diálogo 5+5, dentro de la lógica de ampliación de temas de cooperación que este foro ha tenido a lo largo de su historia, con iniciativas que hemos visto son muy diversas pero siempre en ámbitos vinculados con "la seguridad común, la cooperación económica y el intercambio humano y cultural" $^{35}$, las grandes áreas de trabajo contempladas para esta cooperación.

Dentro del marco del Diálogo 5+5, el objetivo de la nueva Iniciativa 5+5 Defensa era extender al campo de la seguridad y la defensa una fórmula de diálogo y cooperación subregional que ha venido funcionando con relativa continuidad desde su relanzamiento en el año 2001, en un contexto en el que las iniciativas de diálogo y cooperación en la región mediterránea no se han podido mantener en el tiempo con la actividad y el éxito necesarios, pese a ser más ambiciosas y transmitir la imagen de que el $5+5$ podía hacerse "superfluo e innecesario" ${ }^{36}$

La idea de establecer un foro de cooperación regional sobre temas de defensa, que intentara llevar adelante lo que no era posible entonces con los 35 participantes del Proceso de Barcelona, data de septiembre de 2003, a partir de una propuesta del ministro de Defensa

\footnotetext{
${ }^{32}$ Montlaur, Bénédicte: "La France, pays membre du dialogue 5+5", en FMES (2014): Le dialogue 5+5: levier au service... ", op. cit., p. 38. Ver también la p. 40, donde se manifiesta contraria a la extensión de la iniciativa hacia el sur, hacia el Sahel, aunque su experiencia pudiera ser de interés en otras iniciativas futuras en esa región.

${ }^{33}$ Ver, por ejemplo Florensa, Senén: "Mecanismo mediterráneo de cooperación reforzada...", op. cit., p. 34.

${ }^{34}$ Ver Coustillière, Jean François (2012) : "L’initiative 5+5 face au nouveau contexte...", op. cit., p. 7.

35 Romeo, Miguel Ángel (2012): Iniciativa 5+5. Seguridad mediterránea: seguridad compartida, IEEE, Documento Marco 07/2012, p. 6, en http://www.ieee.es/Galerias/fichero/docs marco/2012/DIEEEM07-2012 5x5 SegMed RomeoNunez.pdf

${ }^{36}$ Busquets, Gabriel (2014): "Regional Integration in the Western...", op. cit., p 89.
} 
francés ${ }^{37}$. Dicha idea apostaba por tres características: "un número reducido de países, una zona geográfica limitada al Mediterráneo Occidental" y "proyectos seleccionados en asociación a partir de intereses identificados como comunes" ${ }^{38}$.

Como señala Coustillière, la filosofía de esta idea se basaba en el pragmatismo y en su carácter progresivo, con el objetivo a largo plazo de crear "una dinámica de concertación y de intercambios en el Mediterráneo occidental" ${ }^{39}$. Aunque se planteaba la posibilidad de compartir esta experiencia con el resto de los socios del Proceso de Barcelona, se vio rápidamente que para garantizar sus posibilidades de éxito la iniciativa debía abrirse originalmente a los miembros del grupo 5+5. En julio de 2004 Francia propuso la nueva iniciativa en formato 4+3 -Portugal, España, Francia e Italia más Marruecos, Argelia y Túnez, pero pronto fue ampliada al formato $5+5$ con las incorporaciones de Malta, Libia y Mauritania $^{40}$. Lo que se pretendía en realidad "era establecer una asociación únicamente entre diez países, seleccionando de forma conjunta acciones prácticas a desarrollar que respondieran a necesidades identificadas como comunes y en un espacio geográfico limitado en el que no existían tensiones importantes" ${ }^{41}$. Se ven claramente en este planteamiento algunos de los rasgos esenciales que caracterizarán la iniciativa, y donde radicará en buena medida su continuidad posterior, siempre considerando sus ambiciones limitadas y modestas en las que tanto sus promotores como los analistas coinciden ${ }^{42}$.

\subsection{Los fundamentos de la Iniciativa 5+5 Defensa}

Al igual que los otros ámbitos de cooperación del Diálogo 5+5 de los que antes hablamos, la Iniciativa de Defensa asienta su trabajo en unos principios que, según todos los expertos, son la razón principal de su continuidad en el tiempo y de que haya sobrevivido a las grandes convulsiones que han azotado la región en los últimos años.

El primero de sus fundamentos será la estructura y carácter informal de la Iniciativa. No se trata de una nueva organización, ni se desarrollan nuevas estructuras permanentes de entidad importante, sino que la Iniciativa se pone en marcha a partir de una declaración de intenciones de los diez países participantes - presentada por España y firmada en Paris el 21 de diciembre de 2004- ${ }^{43}$, en la que los Ministros de Defensa establecen unas bases mínimas y muy abiertas para "desarrollar una cooperación multilateral dirigida a promover la seguridad en el Mediterráneo Occidental"44. En la declaración las partes asumieron como principal compromiso que cada país organice una reunión anual de los Ministros de Defensa, de forma rotatoria. Estas reuniones son la oportunidad para discutir, al más alto nivel ministerial, los temas seguridad y defensa que afectan a la región y que forman parte de las preocupaciones compartidas por todos los miembros en cada momento, de igual a igual. La Iniciativa encuentra en estas reuniones su primera utilidad como foro de diálogo e intercambio de opiniones en temas que los participantes han identificado como de interés común y que no son fáciles de tratar en otros formatos multilaterales.

\footnotetext{
${ }^{37}$ Coustillière, Jean François (2007): "La Iniciativa 5+5 Seguridad y Defensa", La Seguridad Multidimensional. $V$ Seminario Internacional sobre Seguridad y Defensa en el Mediterráneo, Barcelona, CIDOB, p. 39, en www.cidob.org/media2/publicacions/monografias/v_seminario seguridad defensa/06 coustillier.

${ }^{38} \mathrm{Ibid}$.

${ }^{39}$ Ibid.

${ }^{40}$ Romeo, op. cit. p. 14.

${ }^{41}$ Coustillière, Jean François (2007): "La Iniciativa 5+5...", op.cit., p. 40.

${ }^{42}$ Coustillière, Jean François (2012) : "L’initiative 5+5 face au nouveau contexte...", op. cit., p. 4.

${ }^{43}$ Romeo, op. cit. p. 14.

${ }_{44}$ Declaración de Intenciones de París, Iniciativa 5+5, 21 de diciembre de 2004, p 1 . en http://www.defensa.gob.es/5mas5/es/doc/declaracion-intenciones-ministros-2004.pdf.
} 
La reunión ministerial tendrá también como objetivo hacer una evaluación de lo realizado en el año anterior, así como aprobar el denominado Plan de Acción anual para el siguiente periodo, que será donde se recojan "aquellas acciones prácticas que en materia de seguridad se desarrollarán a lo largo del año" y que son la parte fundamental de la Iniciativa. La supervisión de este Plan de Acción y la dirección de su ejecución están a cargo de un Comité Director con representantes de los respectivos ministerios, que se reunirá dos veces al año y que designará comités de expertos ad hoc para cada actividad concreta, cuando se precise.

Estas acciones concretas, como también se señala en la declaración de intenciones, son el objeto principal de la Iniciativa: "actividades prácticas de cooperación en aquellos campos de interés común que servirán como punto de encuentro para intercambiar conocimientos y experiencias" 45 . Aquí encontraremos otro de los rasgos esenciales de la Iniciativa que antes citamos, su pragmatismo o ambición limitada, que como señala Echeverría, será el rasgo que "ha caracterizado históricamente a la Iniciativa $5+5$ en su aproximación más general" ${ }^{46}$. En ningún momento se piensa en grandes propuestas políticas de cooperación, grandes acuerdos, grandes ejercicios o proyectos comunes que impliquen un elevado gasto y compromiso político de todos y cada uno de los participantes. La filosofía es bien distinta; acciones de cooperación muy concretas, relativamente sencillas y realizables a corto plazo ${ }^{47}$, con objetivos y ámbitos de interés común para aquellos que deciden participar, que pueden parecer modestas y de poco alcance a un observador ajeno, pero que cumplen perfectamente la función de favorecer "el conocimiento mutuo como medida de confianza y que nos permita abordar asuntos relativos a la seguridad", según señalaba la declaración de París.

Este pragmatismo supone a la vez mantener una importante capacidad de flexibilidad, de manera que la Iniciativa permite ir aumentando los ámbitos de cooperación, siempre que se desarrollen sobre aspectos de interés común de los participantes que en cada momento decidan tomar parte en ellas, sin que además se cuestionen con esta Iniciativa las relaciones y acciones bilaterales que quieran mantener los firmantes. En cada acción concreta que se desarrolla en el marco de la Iniciativa participa quién quiere y tiene interés en hacerlo, sin ninguna obligación para los demás. En este sentido, ya el primer Plan de Acción para la Iniciativa 5+5, anexo a la Declaración de París, recogía los ámbitos de cooperación multilateral iniciales, pero partiendo de que tal cooperación era susceptible de ampliarse a otros campos ${ }^{48}$. Estas áreas de trabajo inicialmente fueron tres y se ampliaron a cuatro posteriormente, esto es: Seguridad Marítima, Seguridad Aérea, participación de las Fuerzas Armadas en la Protección Civil y, añadida más tarde, la Formación y la Investigación en estos temas. Todas las acciones en estos ámbitos se planteaban como cooperación operativa, lo que ha llevado a considerar a este diálogo en Defensa como un marco de cooperación "más técnico que político"49, lo que también explica su permanencia y actividad en los últimos años, levantando menos recelos que otras iniciativas con un mayor compromiso político.

\footnotetext{
${ }^{45}$ Ibid, p. 2.

${ }^{46}$ Echeverría Jesús, Carlos (2012) : "La Iniciativa 5+5 Defensa Sobrevive a las revueltas árabes en 2011", Informe SEDMED, $n^{\circ} 3$, en http://www.sedmed.org/analisis_ssm/documents/informesedmed/echeverria_carlos.pdf.

${ }^{47}$ Coustillière, Jean François (2007): "La Iniciativa 5+5...", op.cit., p. 41.

${ }^{48}$ Plan de Acción para la Iniciativa 5+5. Anexo a la Declaración de Intenciones de París, Iniciativa 5+5, 21 de diciembre de 2004, p. 1, en http://www.defensa.gob.es/5mas5/es/doc/declaracion-intenciones-ministros-2004.pdf.

49 Bellamine, Yassine (2014) : "Le dialogue 5+5 et l'initiative défense : discussions de salon ou actions concrètes? ", p. 3, en http://nawaat.org/portail/2014/12/31/le-dialogue-55-et-linitiative-defense-discussions-desalon-ou-actions-concretes/.
} 
En suma, el carácter informal del dialogo, con una arquitectura "ligera" ${ }^{50}$ que no burocratiza ni complica en exceso la cooperación de los participantes, su pragmatismo al focalizarse en actividades sencillas y prácticas de interés común, unido a la flexibilidad de esta cooperación multilateral, en el que el nivel de compromiso y las acciones concretas los definen los participantes a partir de intereses compartidos, en conjunto, han permitido la continuidad de este diálogo. De este modo, la Iniciativa 5+5 Defensa se ha mantenido en el tiempo, ha progresado y crecido, cumpliendo así con su objetivo inicial de favorecer la confianza y el conocimiento mutuo, precisamente en unos ámbitos -la seguridad y la defensadonde el dialogo y la cooperación de los países europeos y del norte de África presenta tantas dificultades, desconfianza y desencuentros, incluso a la hora de identificar intereses compartidos. Teniendo en cuenta su ambición limitada y su modestia, tanto en objetivos como en medios, el balance de la "cesta" de defensa del Diálogo 5+5, se puede catalogar como razonablemente exitosa.

\section{La Iniciativa 5+5 Defensa en tiempos recientes: La Presidencia española 2014}

La Presidencia española durante 2014 de la Iniciativa 5+5 Defensa, que le correspondía a este país por turno rotatorio, nos permitirá repasar el "estado de salud" de este foro de diálogo e identificar aquellos ámbitos de cooperación multilateral donde los participantes están centrando su atención en la actualidad, una década después de su creación. La organización por parte española, sin que se pretenda hacer aquí un análisis y valoración de las actividades promovidas y sus resultados, nos permitirá ayudar a reconocer las líneas de acción en las que se está moviendo la Iniciativa, el tipo de acciones concretas que se desarrollan en este foro de diálogo y cooperación, así como poder examinar las posibilidades que aparecen cara al futuro en esa búsqueda de campos de interés común, algunas de las cuales han tenido continuidad en la posterior presidencia tunecina de 2015 .

Como señalamos más arriba, la Iniciativa 5+5 Defensa en sus inicios identificó tres ejes fundamentales de interés común donde se podían encontrar aquellas acciones específicas que serían objeto de la cooperación multilateral, concretamente, a) la seguridad marítima, b) la seguridad aérea y c) la cooperación en cuestiones de defensa/protección civil en casos de catástrofe o crisis humanitarias. Posteriormente se incluyó un cuarto ámbito dedicado a d) la formación de personal civil y militar en todos estos campos ${ }^{51}$. Estos son los ámbitos en los que se han venido definiendo los Planes de Acción Anuales, pensados para el corto plazo, en los que se han ido planteando las correspondientes propuestas de colaboraciones concretas, sencillas y realizables en cada periodo, como las que más adelante detallaremos.

Adicionalmente, e independientemente de estos planes anuales ${ }^{52}$, la Iniciativa ha puesto en marcha los denominados Proyectos de Largo Alcance. En este caso, se trata también de iniciativas específicas de cooperación en aspectos o proyectos muy concretos, pero con proyección en el medio y largo plazo, que nacen con vocación de dar continuidad y permanencia a la cooperación entre los países miembros. En la actualidad, los 5 proyectos de largo alcance son los siguientes ${ }^{53}$ :

\footnotetext{
${ }^{50}$ Coustillière, Jean François (2007): "La Iniciativa 5+5...", op.cit., p. 41.

${ }^{51}$ Romeo, op. cit. p. 15.

52 Ibid.

${ }^{53}$ Ministerio de Defensa (2014): Iniciativa 5+5 Defensa. Dossier de Prensa, Oficina de Comunicación, en http://www.defensa.gob.es/5mas5/es/index.html. Sobre los inicios y evolución de estos proyectos ver también Echeverría Jesús, Carlos (2011): "La Iniciativa de Defensa 5+5 avanza a buen ritmo", Informe SEDMED, $\mathrm{n}^{\circ} 1$, pp. 3-5, en http://www.sedmed.org/analisis_ssm/documents/informesedmed/echevarria_jesus.pdf y, sobre sus
} 
1. El Centro Virtual de Control de Tráfico Marítimo Regional (V-RMTC), promovido por Italia y con sede en ese país.

2. El Colegio 5+5 Defensa, de iniciativa francesa, con sede en Toulon, delegaciones en todos los participantes, y en el que España participa a través del CESEDEN, organizando actividades formativas.

3. El Centro Euro-magrebí de Investigación y Estudios Estratégicos para el Mediterráneo Occidental CEMRES, con sede en Túnez.

4. La contribución de las Fuerzas Armadas a la protección civil en caso de catástrofes naturales

5. La cooperación en materia de búsqueda y salvamento marítimo.

En estos marcos que acabamos de señalar, el programa de la presidencia española centró sus actividades en torno a tres pilares ${ }^{54}$ :

1.- el acercamiento de la Iniciativa a la sociedad civil, organizando actividades de carácter académico, con los medios de comunicación, y actividades de divulgación entre los ciudadanos.

2.- Un segundo pilar dirigido a poner en marcha "un sistema de gestión de catástrofes y emergencias", que sirva para compartir las experiencias adquiridas por los participantes en estos ámbitos, para colaborar en la formación del personal y para mejorar la interoperabilidad en la atención sanitaria a los afectados.

3.- El tercer pilar sería el desarrollo de los proyectos en el ámbito de la seguridad marítima y la cooperación ante catástrofes naturales, donde la Iniciativa $5+5$ ya tiene actividad pero que se puede abrir a otras iniciativas o coordinar mejor con otros ámbitos del Diálogo $5+5$ con los que pueden coincidir en algunos aspectos.

En este sentido, las actividades concretas de la presidencia española, además de las reuniones del Comité Director y de los Jefes de Estado Mayor, consistieron en un seminario sobre "Perspectivas de Genero en las Fuerzas Armadas", un seminario general de la Presidencia sobre el estado de la Iniciativa, un seminario de orientación fundamentalmente académica en el CEU, unas jornadas sobre Gestión de desastres naturales en el Mediterráneo Occidental a cargo de la Unidad Militar de Emergencias UME y la organización de dos de los tradicionales ejercicios o maniobras aeronavales de la Iniciativa, concretamente el ejercicio "Seaborder 2014" sobre seguridad marítima y la participación en el "Circaete 2014" sobre seguridad aérea. La reunión anual de Ministros de Defensa de la Iniciativa, celebrada en Granada, además de repasar lo hecho durante la presidencia española y trabajar sobre el Plan de Acción 2015, que se está llevando a cabo bajo la presidencia de Túnez, fue la oportunidad para identificar e intercambiar opiniones sobre las que son en la actualidad las principales preocupaciones compartidas a ambos lados del Mediterráneo, susceptibles de acciones de cooperación futura: lucha contra el terrorismo yihadista, inmigración ilegal, crimen organizado, y cooperación en materia de ciberdefensa (a propuesta española) ${ }^{55}$.

desarrollos más recientes, ver Adam, Philippe:"Les diferentes dimensions et actions du dialogue 5+5" en FMES (2014): "Le dialogue 5+5: levier au service..." , op. cit., pp. 60-66.

${ }^{54}$ Ministerio de Defensa (2014): Iniciativa 5+5 Defensa. Dossier de Prensa..., op. cit., p. 4.

${ }^{55}$ Talironte, Elena (2015): "Todos juntos contra el yihadismo", en Revista Española de Defensa, enero 2015 , p. 24. 
Paralelamente a las actividades organizadas por España como país que ostentaba la presidencia, cumpliendo así con ese principio de flexibilidad del que hablamos, otros participantes contribuyeron a la Iniciativa $5+5$ organizando sus propias actividades ${ }^{56}$. Así, sin ánimo de ser exhaustivo, Argelia organizó un seminario sobre medios aéreos y catástrofes naturales, varios países organizaron módulos de formación del Colegio $5+5$ en el que participaron alumnos de todos los países miembros, Túnez acogió la reunión del Comité del Centro Euro-magrebí de Investigación y Estudios Estratégicos (CEMRES) y varios países se reunieron en Italia para tratar sobre el Centro Virtual de Control del Tráfico Marítimo Regional y en Malta para dar impulso a la Web de la Iniciativa. En el ámbito de las maniobras militares enmarcados en la Iniciativa 5+5, Marruecos organizó los ejercicios "Tammaris 2014" sobre búsqueda y salvamento y "Simulex 2014" sobre contaminación marítima, en los que participaron varios de los países de la Iniciativa.

En definitiva, toda esta serie de actividades, organizadas por España y por otros países participantes, son una buena muestra del tipo de acciones de cooperación que el marco de la Iniciativa 5+5 permite; acciones no muy complejas, sin grandes despliegues de medios, pero que permiten que en un ejercicio como Seaborder 2014, por ejemplo, participen juntos fuerzas navales de Portugal, España, Marruecos y Argelia, países que tienen intereses contrapuestos en distintas cuestiones e incluso malas relaciones y conflictos abiertos en algunos casos. En suma, estas actividades concretas en las que colaboran los países europeos con los africanos y los africanos entre ellos, responden perfectamente a la filosofía y a los principios que mueven la iniciativa. Además de dar visibilidad publica a este foro de cooperación multilateral y proyectarlo como un mecanismo para reforzar las relaciones de los países del Mediterráneo Occidental, todas ellas son oportunidades para reunir a representantes de los distintos miembros, favorecer el trabajo en conjunto, el intercambio de ideas y experiencias, compartir percepciones $\mathrm{y}$, en definitiva, promover el conocimiento mutuo. Como señala algún autor "¿Acaso la dinámica de las medidas de confianza no radica en eso?" ${ }^{57}$.

\section{Conclusiones: la Iniciativa 5+5 en el contexto de la agenda de seguridad europea y sus perspectivas de futuro}

Las relaciones entre los países del norte de África y los estados Europeos no han sido fáciles en las últimas décadas ${ }^{58}$. El Mediterráneo es un espacio tan amplio y diverso como complejo, y las iniciativas para reforzar el diálogo y las relaciones entre los vecinos de uno y otro lado se han encontrado habitualmente con los problemas derivados de esta complejidad, llevándolas a desarrollarse con un perfil muy bajo cuando no al estancamiento o el fracaso.

En el caso de las cuestiones de seguridad y defensa, la situación ha sido, si cabe, aún peor. Las visiones y las percepciones entre el norte y el sur del Mediterráneo -y entre los mismos países del sur- han sido tradicionalmente muy distintas y enfrentadas. Si hay una palabra que las puede definir, es la desconfianza.

En este contexto vimos que aparecería en los 90 el Diálogo $5+5$ y, específicamente sobre Seguridad y Defensa, la Iniciativa 5+5 Defensa a partir de 2004. En ningún momento la Iniciativa de Defensa nació con la vocación de sustituir a ningún foro de cooperación sobre

\footnotetext{
${ }^{56}$ Ibid.

${ }^{57}$ Coustillière, Jean François (2007): "La Iniciativa 5+5... " , op.cit., p. 44.

${ }^{58}$ Para un recorrido y valoración de la situación política general del Mediterráneo en los últimos años y en la actualidad ver, Bergamini, Patrice (2014): "La situation en Méditerranée et les derniers développments en matière de relations euroméditerranéennes", en FMES (2014): "Le dialogue 5+5: levier au service..." , op.cit., pp. 23-34.
} 
estos temas de mayor alcance (las relaciones entre la UE y el Norte de África, por ejemplo, o la Unión por el Mediterráneo), sino que su área de acción aparece desde el principio circunscrito al Mediterráneo Occidental, limitando tanto el alcance geográfico como el número de socios participantes, los diez del Diálogo. Esta voluntad de limitación, que algunos ven como una debilidad de la iniciativa, seguramente es la primera de las razones de su continuidad. Manteniendo esta iniciativa como un foro claramente subregional ha permitido gestionar mejor sus dinámicas y aislarla -aunque no siempre-, de los graves conflictos de la región. El conflicto en Libia de los últimos años es la excepción, pero incluso en este caso el foro se ha convertido en un instrumento potencialmente relevante para el intercambio de opiniones y el diálogo abierto sobre un tema de tanta importancia para todos sus miembros ${ }^{59}$.

Difícilmente la Iniciativa funcionaría mejor con un número mayor de miembros (ya hay otros foros para intentarlo), pero la experiencia de mantener esa orientación subregional para favorecer el contacto y el conocimiento mutuo parece que debe tenerse en cuenta cara a otros procesos. Más aún cuando se trata de temas de tanta sensibilidad como las cuestiones de Defensa y Seguridad. La iniciativa en modo alguno interfiere en las relaciones bilaterales que tienen los participantes entre ellos, sino que las complementa e incluso las facilita, como ocurre en el caso de Marruecos y Argelia. Tampoco aspira, ni está preparada o diseñada para ello, para sustituir a otros foros multilaterales de cooperación más estructurada, sino que pretende acercar a los participantes para que la colaboración en otros ámbitos sea más sencilla. En este sentido, el Diálogo en general y la Iniciativa de Defensa en particular se muestran como instrumentos útiles para que las diferencias de valores y de identidad entre los miembros del norte y del sur -que existen-, no sean impedimento a la hora de profundizar en la cooperación con el norte de África en temas sensibles. Esta forma de diálogo parece más adecuada a la hora de hacer frente y competir con la influencia que otros países árabes del Este del Mediterráneo -con los que sí comparten valores e identidad- están intentando proyectar sobre los países de la subregión.

El formato $5+5$ ha permitido también mantener otra de las características que está detrás de la continuidad de la iniciativa, la relación equilibrada y de igual a igual. En la Iniciativa 5+5 nada se impone a los participantes. Los temas objeto de discusión y cooperación son aquellos que todos identifican como comunes y compartidos, y los países deciden en cuáles de las acciones de cooperación (maniobras, proyectos, cursos de formación, seminarios, reuniones, grupos de trabajo, etc.) participan y en cuáles no, permaneciendo abiertas a todos. La arquitectura organizativa es mínima. Esta libertad y flexibilidad que proporciona el marco $5+5$ ha demostrado ser muy positiva y favorecer la proliferación de iniciativas en las que antes o después los países se encuentran y mantienen contacto, cumpliendo así su objetivo de favorecer el conocimiento y la confianza mutua, que desde sus orígenes ha sido sin duda el objetivo esencial de este foro.

La orientación "gradualista" o "incrementalista" del formato, que parte de unas bases muy modestas, pero permite ir aumentando los ámbitos y las acciones de cooperación según todos los miembros estén dispuestos a hacerlo, también ha demostrado ser adecuada. El Diálogo 5+5 y dentro del mismo la Iniciativa de Defensa son sin duda iniciativas limitadas en cuanto a sus objetivos, pero precisamente por ello se puede hablar de su cumplimiento y, por tanto, de su éxito, lo que está en línea con nuestra hipótesis inicial. El crecimiento controlado que permite esta formula de cooperación parece ser otro de sus aciertos, tal vez exportable a otras iniciativas. Un intento de potenciación excesivo de la Iniciativa, buscando llevarla rápidamente a niveles de compromiso muy superiores, muy probablemente sólo encontraría el

\footnotetext{
${ }^{59}$ Adam, Philippe:"Les diferentes dimensions et actions du dialogue 5+5" en FMES (2014): "Le dialogue 5+5: levier au service...", op.cit., p. 58.
} 
rechazo de los miembros del sur y su caída en el olvido y la irrelevancia absoluta. De ahí su interés en mantenerla con su filosofía actual, y creciendo sólo cuando sea posible, sirviendo de instrumento potenciador y facilitador de las relaciones, del que se beneficiarían otras iniciativas de diálogo y concertación de mayor ambición política en las que se está trabajando. A su vez, todo ello permite dar una perspectiva adecuada sobre sus grandes limitaciones a la hora de construir una asociación entre el norte y el sur del Mediterráneo.

La orientación práctica de la Iniciativa 5+5 Defensa presenta muchas ventajas en el actual contexto de seguridad en el Mediterráneo. Buena parte de los grandes desafíos e incluso amenazas que afectan a la región en los últimos años forman parte de los ámbitos de cooperación objeto de la Iniciativa $5+5$ defensa desde sus orígenes hace una década. La seguridad marítima, el tráfico de personas en el Mediterráneo y las muertes que se producen como consecuencia de la falta de seguridad en las travesías, el crimen organizado, los problemas de la inmigración ilegal, la respuesta ante catástrofes naturales y, más recientemente el auge del terrorismo yihadista en la región, son algunos de los problemas más acuciantes en la región y a los que se está buscando respuesta en los países europeos y en el norte de África. Sobre varios de estos aspectos ya han existido experiencias de trabajo conjunto dentro del marco general y de la Iniciativa de Defensa en particular, y las posibilidades de incrementar el nivel de cooperación parecen lógicas y no se deberían desaprovechar. Otros foros del Dialogo 5+5 como las reuniones de Ministros de Asuntos Exteriores, Interior o Asuntos Sociales han tenido de forma habitual en la agenda los temas de la seguridad y de las migraciones ilegales ${ }^{60}$, y las implicaciones de estos asuntos desde el punto de vista de la seguridad y la defensa parecen evidentes.

Hay por lo tanto un amplio espacio de cooperación sobre estos temas de la "nueva agenda", fundado en esa flexibilidad que el Dialogo $5+5$ y su Iniciativa de Defensa han demostrado. En ese sentido las dinámicas creadas en esas actividades realizadas en común pueden ser muy útiles para incrementar el nivel de colaboración entre las dos orillas, para favorecer el intercambio de información y para buscar soluciones conjuntas o colaborativas basadas en la confianza mutua- que parecen imprescindibles. La aproximación "técnica" a estos problemas que tienen tanto el Diálogo como la Iniciativa de Defensa, con mucho menor peso de la discusión política de alto nivel (aunque existe), facilita el tratamiento de estos asuntos, donde la diversidad de percepciones a la hora de diagnosticarlos y encontrar soluciones es muy importante entre los países participantes.

En definitiva, aunque se puede afirmar que el Diálogo $5+5$ y la Iniciativa $5+5$ Defensa tienen una salud razonablemente buena (dentro de sus niveles de ambición), parece claro que su futuro depende de que mantenga sus características fundamentales, lo que está de acuerdo con nuestra hipótesis inicial. La Iniciativa 5+5 Defensa cubre un hueco que ninguna otra iniciativa multilateral ha conseguido llenar, pero pensar en ella como el embrión de una cooperación estructurada de mucho mayor calado en la región del Mediterráneo Occidental e incluso extenderla al conjunto del Mediterráneo parece absolutamente ilusorio. Lo mismo podríamos decir del Diálogo $5+5$ en general. Las posibilidades de crecimiento y profundización que permiten su diseño y su filosofía son grandes, básicamente hasta dónde sus participantes quieran llegar, pero no parece razonable pensar en el Diálogo y en la Iniciativa como los instrumentos para conseguir grandes y complejas estructuras de cooperación que solucionen ni los problemas de esta subregión ni los problemas internos de los países miembros, ya sean del norte o del sur del Mediterráneo. Sus logros en medidas y mecanismos concretos y su carácter facilitador, como mecanismo de fomento de la confianza, son tal vez sus mejores logros y en absoluto tienen que ser minusvalorados por ello.

\footnotetext{
${ }^{60}$ Ben M’Barek, Slaheddine (2009): "La cooperation en Mediterranee...", op. cit., p. 50.
} 